\title{
Iterative guided image fusion
}

\author{
Alexander Toet ${ }^{\text {Corresp. } 1}$ \\ ${ }^{1}$ TNO, Soesterberg, Netherlands \\ Corresponding Author: Alexander Toet \\ Email address: lextoet@gmail.com
}

We propose a multi-scale image fusion scheme based on guided filtering. Guided filtering can effectively reduce noise while preserving detail boundaries. When applied in an iterative mode, guided filtering selectively eliminates small scale details while restoring larger scale edges. The proposed multi-scale image fusion scheme achieves spatial consistency by using guided filtering both at the decomposition and at the recombination stage of the multi-scale fusion process. First, size-selective iterative guided filtering is applied to decompose the source images into approximation and residual layers at multiple spatial scales. Then, frequency-tuned filtering is used to compute saliency maps at successive spatial scales. Next, at each spatial scale binary weighting maps are obtained as the pixelwise maximum of corresponding source saliency maps. Guided filtering of the binary weighting maps with their corresponding source images as guidance images serves to reduce noise and to restore spatial consistency. The final fused image is obtained as the weighted recombination of the individual residual layers and the mean of the approximation layers at the coarsest spatial scale. Application to multiband visual (intensified) and thermal infrared imagery demonstrates that the proposed method obtains state-of-the-art performance for the fusion of multispectral nightvision images. The method has a simple implementation and is computationally efficient. 
1

2

3

4

5

6

7

8

9

10

11

\section{Iterative Guided Image Fusion}

\author{
Alexander Toet \\ TNO \\ Kampweg 5, 3769DE, Soesterberg, The Netherlands \\ E lex.toet@tno.nl
}




\section{Introduction}

14

The increasing deployment and availability of co-registered multimodal imagery from different types of sensors has spurred the development of image fusion techniques. The information provided by different sensors registering the same scene can either be (partially) redundant or complementary and may be corrupted with noise. Effective combinations of complementary and partially redundant multispectral imagery can therefore visualize information that is not directly evident from the individual input images. For instance, in nighttime (low-light) outdoor surveillance applications, intensified visual (II) or near-infrared (NIR) imagery often provides a detailed but noisy representation of a scene. While different types of noise may result from several processes associated with the underlying sensor physics, additive noise is typically the predominant noise component encountered in II and NIR imagery (Petrovic \& Xydeas 2003). Additive noise can be modelled as a random signal that is simply added to the original signal. As a result, additive noise may obscure or distort relevant image details. In addition, targets of interest like persons or cars are sometimes hard to distinguish in II or NIR imagery because of their low luminance contrast. While thermal infrared (IR) imagery typically represents these targets with high contrast, their background (context) is often washed out due to low thermal contrast. In this case, a fused image that clearly represents both the targets and their background enables a user to assess the location of targets relative to landmarks in their surroundings, thus providing more information than either of the input images alone.

Some potential benefits of image fusion are: wider spatial and temporal coverage, decreased uncertainty, improved reliability, and increased system robustness. Image fusion has important applications in defense and security for situational awareness (Toet et al. 1997), surveillance (Shah et al. 2013; Zhu \& Huang 2007), target tracking (Motamed et al. 2005; Zou \& Bhanu 2005), intelligence gathering (O'Brien \& Irvine 2004), concealed weapon detection (Bhatnagar \& Wu 2011; Liu et al. 2006; Toet 2003; Xue \& Blum 2003; Xue et al. 2002; Yajie \& Mowu 2009), detection of abandoned packages (Beyan et al. 2011) and buried explosives (Lepley \& Averill 2011), and face recognition (Kong et al. 2007; Singh et al. 2008). Other important image fusion applications are found in industry (Tian et al. 2009), art analysis (Zitová et al. 2011), agriculture (Bulanona et al. 2009), remote sensing (Ghassemian 2001; Jacobson \& Gupta 2005; Jacobson et al. 2007; Jiang et al. 2011) and medicine (Agarwal \& Bedi 2015; Biswas et al. 2015; Daneshvar \& Ghassemian 2010; Singh \& Khare 2014; Wang et al. 2014; Yang \& Liu 2013) (for a survey of different applications of image fusion techniques see (Blum \& Liu 2006)).

In general, image fusion aims to represent the visual information from any number of input images in a single composite (fused) image that is more informative than each of the input images alone, eliminating noise in the process while preventing both the loss of essential information and the introduction of artefacts. This requires the availability of filters that combine the extraction of relevant image details with noise reduction.

To date, a variety of image fusion algorithms have been proposed. A popular class of algorithms are the multi-scale image fusion schemes, which decompose the source images into spatial primitives at multiple spatial scales, then integrate these primitives to form a new ('fused') multi- 
scale representation, and finally apply an inverse multi-scale transform to reconstruct the fused image. Examples of this approach are for instance the Laplacian pyramid (Burt \& Adelson 1983), the Ratio of Low-Pass pyramid (Toet 1989b), the contrast pyramid ((Toet et al. 1989)), the filter-subtract-decimate Laplacian pyramid (Burt 1988; Burt \& Kolczynski 1993), the gradient pyramid (Burt 1992; Burt \& Kolczynski 1993), the morphological pyramid (Toet 1989a), the discrete wavelet transform (Lemeshewsky 1999; Li et al. 1995; Li et al. 2002; Scheunders \& De Backer 2001), the shift invariant discrete wavelet transform (Lemeshewsky 1999; Rockinger 1997; Rockinger 1999; Rockinger \& Fechner 1998), the contourlet (Yang et al. 2010), the shift-invariant shearlet transform (Wang et al. 2014), the non-subsampled shearlet transform (Kong et al. 2015; Liu et al. 2016; Zhang et al. 2015), the ridgelet transform (Tao et al. 2005). The filters applied in several of the earlier techniques typically produce halo artefacts near edges. More recent methods like shearlets, contourlets and ridgelets are better capable to preserve local image features but are often complex or time-consuming.

Non-linear edge-preserving smoothing filters such as anisotropic diffusion (Perona \& Malik 1990), robust smoothing (Black et al. 1998) and the bilateral filter (Tomasi \& Manduchi 1998) may appear effective tools to prevent artefacts that arise from spatial inconsistencies in multiscale image fusion schemes. However, anisotropic diffusion tends to over sharpen edges and is computationally expensive, which makes it less suitable for application in multi-scale fusion schemes (Farbman et al. 2008). The non-linear bilateral filter (BLF) assigns each pixel a weighted mean of its neighbors, with the weights decreasing both with spatial distance and with difference in value (Tomasi \& Manduchi 1998). While the BLF is quite effective at smoothing small intensity changes while preserving strong edges and has efficient implementations, it also tends to blur across edges at larger spatial scales, thereby limiting its value for application in multi-scale image decomposition schemes (Farbman et al. 2008). In addition, the BLF has the undesirable property that it can reverse the intensity gradient near sharp edges (the weighted average becomes unstable when a pixel has only few similar pixels in its neighborhood: He et al. 2013). In the joint (or cross) bilateral filter (JBLF) a second or guidance image serves to steer the edge stopping range filter thus preventing over- or under- blur near edges (Petschnigg et al. 2004). Zhang et al. (2014) showed that the application of the JBLF in an iterative framework results in size selective filtering of small scale details combined with the recovery of larger scale edges. The recently introduced Guided Filter (GF: He et al. 2013) is a computationally efficient, edge-preserving translation-variant operator based on a local linear model which avoids the drawbacks of bilateral filtering and other previous approaches. When the input image also serves as the guidance image, the GF behaves like the edge preserving BLF. Hence, the GF can gracefully eliminate small details while recovering larger scale edges when applied in an iterative framework.

In this paper we propose a multi-scale image fusion scheme, where iterative guided filtering is used to decompose the input images into approximate and residual layers at successive spatial scales, and guided filtering is used to construct the weight maps used in the recombination process.

The rest of this paper is organized as follows. Section 2 briefly discusses the principles of edge preserving filtering and introduces (iterative) guided filtering. In Section 3 we discuss related work. Section 4 presents the proposed guided fusion based image fusion scheme. Section 5 
103 presents the imagery and computational methods that were used to assess the performance of the 104 new image fusion scheme. The results of the evaluation study are presented in Section 6.

105 Finally, in Section 7 the results are discussed and some conclusions are presented.

106

107

108

109

110

111

112

113

114

115

116

117

118

119

120

121

122

123

124

125

126

127

128

129

130

131

132

133

134

135

136

137

138

139

140

141

142

143

144

145

\section{Edge preserving filtering}

In this section we briefly introduce the edge preserving bilateral and joint bilateral filters, show how they are related to the guided filter, and how the application of a guided filter in an iterative framework results in size selective filtering of small scale image details combined with the recovery of larger scale edges.

\subsection{Bilateral filter}

Spatial filtering is a common operation in image processing that is typically used to reduce noise or eliminate small spurious details (e.g., texture). In spatial filtering the value of the filtered image at a given location is a function (e.g., a weighted average) of the original pixel values in a small neighborhood of the same location. Although low pass filtering or blurring (e.g., averaging with Gaussian kernel) can effectively reduce image noise, it also seriously degrades the articulation of (blurs) significant image edges. Therefore, edge preserving filters have been developed that reduce small image variations (noise or texture) while preserving large discontinuities (edges).

The bilateral filter is a non-linear filter that computes the output at each pixel as a Gaussian weighted average of their spatial and spectral distances. It prevents blurring across edges by assigning larger weights to pixels that are spatially close and have similar intensity values ((Tomasi \& Manduchi 1998)). It uses a combination of (typically Gaussian) spatial and a range (intensity) filter kernels that perform a blurring in the spatial domain weighted by the local variation in the intensity domain. It combines a classic low-pass filter with an edge-stopping function that attenuates the filter kernel weights at locations where the intensity difference between pixels is large. Bilateral filtering was developed as a fast alternative to the computationally expensive technique of anisotropic diffusion, which uses gradients of the filtering images itself to guide a diffusion process, avoiding edge blurring ((Perona \& Malik 1990)). More formally, at a given image location (pixel) $i$, the filtered output $O_{i}$ is given by:

$$
O_{i}=\frac{1}{K_{i}} \sum_{j \in \Omega} I_{j} f(\|i-j\|) g\left(\left\|I_{i}-I_{j}\right\|\right)
$$

where

$$
f \text { is the spatial filter kernel (e.g., a Gaussian centered at } i \text { ), }
$$

$g$ is the range or intensity (edge-stopping) filter kernel (centered at the image value at $i$ ),

$\Omega$ is the spatial support of the kernel,

$K_{i}$ is a normalizing factor (the sum of the $f \cdot g$ filter weights) . 
146 Intensity edges are preserved since the bilateral filter decreases not only with the spatial distance 147 but also with the intensity distance. Though the filter is efficient and effectively reduces noise

148 while preserving edges in many situations, it has the undesirable property that it can reverse the 149 intensity gradient near sharp edges (the weighted average becomes unstable when a pixel has

150 only few similar pixels in its neighborhood: He et al. 2013).

151

152

153

154

155

156

157

158

159

160

161

162

163

164

165

166

167

168

169

170

171

172

173

174

175

176

177

178

179

180

181

182

183

In the joint (or cross) bilateral filter (JBLF) the range filter is applied to a second or guidance image G (Petschnigg et al. 2004):

$$
O_{i}=\frac{1}{K_{i}} \sum_{j \in \Omega} I_{j} \cdot f(\|i-j\|) \cdot g\left(\left\|G_{i}-G_{j}\right\|\right)
$$

The JBLF can prevent over- or under- blur near edges by using a related image $G$ to guide the edge stopping behavior of the range filter. That is, the JBLF smooths the image $I$ while preserving edges that are also represented in the image $G$. The JBLF is particularly favored when the edges in the image that is to be filtered are unreliable (e.g., due to noise or distortions) and when a companion image with well-defined edges is available (e.g., in the case of flash /noflash image pairs).image. Thus, in the case of filtering an II image for which a companion (registered) IR image is available, the guidance image may either be the II image itself or its IR counterpart.

\subsection{Guided filtering}

A guided image filter (He et al. 2013) is a translation-variant filter based on a local linear model. Guided image filtering involves an input image $I$, a guidance image $G$ ) and an output image $O$. The two filtering conditions are $(i)$ that the local filter output is a linear transform of the guidance image $G$ and (ii) as similar as possible to the input image $I$. The first condition implies that

$$
O_{i}=a_{k} G_{i}+b_{k} \quad \forall i \in \omega_{k}
$$

where $\omega_{k}$ is a square window of size $(2 r+1) \times(2 r+1)$. The local linear model ensures that the output image $O$ has an edge only at locations where the guidance image $G$ has one, because $\nabla O=a \nabla G$. The linear coefficients $a_{k}$ and $b_{k}$ are constant in $\omega_{k}$. They can be estimated by minimizing the squared difference between the output image $O$ and the input image $I$ (the second filtering condition) in the window $\omega_{k}$, i.e. by minimizing the cost function $E$ :

$$
E\left(a_{k}, b_{k}\right)=\sum_{i \in \omega_{k}}\left(\left(a_{k} G_{i}+b_{k}-I_{i}\right)^{2}+\varepsilon a_{k}^{2}\right)
$$

where $\varepsilon$ is a regularization parameter penalizing large $a_{k}$. The coefficients $a_{k}$ and $b_{k}$ can directly be solved by linear regression (He et al. 2013):

$$
a_{k}=\frac{\frac{1}{|\omega|} \sum_{i \in \omega_{k}} G_{i} I_{i}-\bar{G}_{k} \bar{I}_{k}}{\sigma_{k}^{2}+\varepsilon}
$$

where

$$
b_{k}=\bar{I}_{k}-a_{k} \bar{G}_{k}
$$

where

$|\omega|$ is the number of pixels in $\omega_{k}$, 
184

185

186

187

188

189

190

192

193

194

195

196

197

198

199

200

201

202

203

204

205

206

207

208

209

210

211

212

213

214

215

216

217

218

219

220

$\bar{I}_{k}$ and $\bar{G}_{k}$ represent the means of respectively $I$ and $G$ over $\omega_{k}$, and $\sigma_{k}^{2}$ is the variance of $I$ over $\omega_{k}$.

Since pixel $i$ is contained in several different (overlapping) windows $\omega_{k}$, the value of $O_{i}$ in Equation (3) depends on the window over which it is calculated. This can be accounted for by averaging over all possible values of $O_{i}$ :

$$
O_{i}=\frac{1}{|\omega|} \sum_{k \mid i \in \omega_{k}}\left(a_{k} G_{k}+b_{k}\right)
$$

Since $\sum_{k \mid i \in \omega_{k}} a_{k}=\sum_{k \in \omega_{i}} a_{k}$ due to the symmetry of the box window Equation (7) can be written as

$$
O_{i}=\bar{a}_{i} G_{i}+\bar{b}_{i}
$$

where $\bar{a}_{i}=\frac{1}{|\omega|} \sum_{k \in \omega_{i}} a_{k}$ and $\bar{b}_{i}=\frac{1}{|\omega|} \sum_{k \in \omega_{i}} b_{k}$ are the average coefficients of all windows overlapping $i$. Although the linear coefficients $\left(\bar{a}_{i}, \bar{b}_{i}\right)$ vary spatially, their gradients will be smaller than those of $G$ near strong edges (since they are the output of a mean filter). As a result we have $\nabla O \approx \bar{a} \nabla G$, meaning that abrupt intensity changes in the guiding image $G$ are still largely preserved in the output image $O$.

Equations (5), (6) and (8) define the guided filter. When the input image also serves as the guidance image, the guided filter behaves like the edge preserving bilateral filter, with the parameters $\varepsilon$ and the window size $r$ having the same effects as respectively the range and the spatial variances of the bilateral filter. Equation (8) can be rewritten as

$$
O_{i}=\sum_{j} W_{i j}(G) I_{j}
$$

with the weighting kernel $W_{i j}$ depending only on the guidance image $G$ :

$$
W_{i j}=\frac{1}{|\omega|^{2}} \sum_{k:(i, j) \in \omega_{k}}\left(1+\frac{\left(G_{i}-\bar{G}_{k}\right)\left(G_{j}-\bar{G}_{k}\right)}{\sigma_{k}^{2}+\varepsilon}\right)
$$

Since $\sum_{j} W_{i j}(G)=1$ this kernel is already normalized.

The guided filter is a computationally efficient, edge-preserving operator which avoids the gradient reversal artefacts of the bilateral filter. The local linear condition formulated by Equation (3) implies that its output is locally approximately a scaled version of the guidance image plus an offset. This makes it possible to use the guided filter to transfer structure from the guidance image $G$ to the output image $O$, even in areas where the input image $I$ is smooth (or flat). This structure- transferring filtering is an useful property of the guided filter, and can for instance be applied for feathering/matting and dehazing (He et al. 2013).

\subsection{Iterative guided filtering}

Zhang et al. (Zhang et al. 2014) showed that the application of the joint bilateral filter (Equation (2)) in an iterative framework results in size selective filtering of small scale details combined with the recovery of larger scale edges. In this scheme the result $G^{t+1}$ of the $t$-th iteration is 
221

222

223

224

225

226

227

228

229

230

231

232

233

234

235

236

237

238

239

240

241

242

243

244

245

246

247

248

249

250

251

252

253

254

255

256

257

258

259

260

261

262

263

264

obtained from the joint bilateral filtering of the input image $I$ using the result $G^{t}$ of the previous iteration step as the guidance image:

$$
G_{i}^{t+1}=\frac{1}{K_{i}} \sum_{j \in \Omega} I_{j} \cdot f(\|i-j\|) \cdot g\left(\left\|G_{i}^{t}-G_{j}^{t}\right\|\right)
$$

In this scheme details smaller than the Gaussian kernel of the bilateral filter are removed while the edges of the remaining details are iteratively restored. Hence, this scheme allows the selective elimination of small scale details while preserving the remaining image structure. Note that the initial guidance image $G^{1}$ can simply be a constant (e.g., zero) valued image since it updates to the Gaussian filtered input image in the first iteration step. Here we propose to replace the bilateral filter in this scheme by a guided filter to avoid any gradient reversal artefacts.

\section{Related work}

As mentioned before most multi-scale transform-based image fusion methods introduce some artefacts because the spatial consistency is not well-preserved (Li et al. 2013). This has led to the use of edge preserving filters to decompose source images into approximate and residual layers while preserving the edge information in the fusion process. Techniques that have been applied include weighted least squares filter (Yong \& Minghui 2014), $\mathrm{L}_{1}$ fidelity using $\mathrm{L}_{0}$ gradient (Cui et al. 2015), $\mathrm{L}_{0}$ gradient minimization (Zhao et al. 2013), cross bilateral filter (Kumar 2013) and anisotropic diffusion (Bavirisetti \& Dhuli 2016a).

Li et al. (2013) proposed to restore spatial consistency by using guided filtering in the weighted recombination stage of the fusion process. In their scheme, the input images are first decomposed into approximate and residual layers using a simple averaging filter. Next, each input image is then filtered with a Laplacian kernel followed by blurring with a Gaussian kernel, and the absolute value of the result is adopted as a saliency map that characterizes the local distinctness of the input image details. Then, binary weight maps are obtained by comparing the saliency maps of all input images, and assigning a pixel in an individual weight map the value 1 if it is the pixelwise maximum of all saliency maps, and 0 otherwise. The resulting binary weight maps are typically noisy and not aligned with object boundaries and may produce artefacts to the fused image. Li et al. (2013) performed guided filtering on each weight map with its corresponding source layer as the guidance image, to reduce noise and to restore spatial consistency. The GF guarantees that pixels with similar intensity values have similar weights and weighting is not performed across edges. Typically a large filter size and a large blur degree are used to fuse the approximation layers, while a small filter size and a small blur degree are used to combine the residual layers. Finally, the fused image is obtained by weighted recombination of the individual source residual layers. Despite the fact that this method is efficient and can achieve state-of-theart performance in most cases, it does not use edge preserving filtering in the decomposition stage and applies a saliency map that does not relate well to human visual saliency (Gan et al. 2015).

In their multi-scale image fusion framework Gan et al. (2015) apply edge preserving filtering in the decomposition stage to extract well-defined image details (i.e., to preserve their edges) and use guided filtering in the weighted recombination stage to reduce spatial inconsistencies introduced by the weighting maps used in the reconstruction stage (i.e., to prevent edge artefacts like halos). First, a nonlinear weighted least squares edge-preserving filter (Farbman et al. 2008) 
265 is used to decompose the source images into approximate and residual layers. Next, phase

266 congruency is used to calculate saliency maps that characterize the local distinctness of the

267 source image details. The rest of their scheme is similar to that of Li et al. (2013): binary weight

268 maps are obtained from pixelwise comparison of the saliency maps corresponding to the

269 individual source images; guided filtering is applied to these binary weight maps to recue noise

270

271

272

273 and restore spatial consistency, and the fused image is obtained by weighted recombination of the individual source residual layers.

274

275

276

277

278

279

280

281

282

283

284

285

286

287

288

289

290

291

292

293

294

295

296

297

298

299

300

301

302

303

304

305

306

307

\section{Proposed method}

A flow chart of the proposed multi-scale decomposition fusion scheme is shown in Figure 1. The algorithm consists of the following steps:

1. Iterative guided filtering is applied to decompose the source images into approximate layers (representing large scale variations) and residual layers (containing small scale variations).

2. Frequency-tuned filtering (Achanta et al. 2009) is used to generate saliency maps for the source images.

3. Binary weighting maps are computed as the pixelwise maximum of the individual source saliency maps.

4. Guided filtering is applied to each binary weighting map with its corresponding source as the guidance image to reduce noise and to restore spatial consistency.

5. The fused image is computed as a weighted recombination of the individual source residual layers.

In a hierarchical framework steps 1-4 are performed at multiple spatial scales. In this paper we used a 4 level decomposition obtained by filtering at 3 different spatial scales (see Figure 1).

Figure 2 shows the intensified visual (II) and thermal infrared (IR) or near infrared (NIR) images together with the results of the proposed image fusion scheme, for the 12 different scenes that were used in the present study. We will now discuss the proposed fusion scheme in more detail.

Consider two co-registered source images $X_{0}(x, y)$ and $Y_{0}(x, y)$. The proposed scheme then applies iterative guided filtering (IGF) to the input images $X_{i}$ and $Y_{i}$ to obtain progressively coarser image representations $X_{i+1}$ and $Y_{i+1}(i>0)$ :

$$
\operatorname{IGF}\left(X_{i}, r_{i}, \varepsilon_{i}\right)=X_{i+1} \quad ; \quad i \in\{0,1,2\}
$$

where the parameters $\varepsilon_{i}$ and $r_{i}$ represent respectively the range and the spatial variances of the guided filter at iteration step $i$. In this study the number of iteration steps is set to 4 . By letting each finer scale image serve as the approximate layer for the preceding coarser scale image the successive size-selective residual layers $d X_{i}$ are simply obtained by subtraction as follows:

$$
d X_{i}=X_{i}-X_{i+1} \quad ; \quad i \in\{0,1,2\}
$$

Figure 3 shows the approximate and residual layers that are obtained this way for the tank scene (nr 10 in Figure 2). The edge-preserving properties of the iterative guided filter guarantee a graceful decomposition of the source images into details at different spatial scales. The filter size 
308 and regularization parameters used in this study are respectively set to $r_{i}=\{5,10,30\}$ and

$309 \varepsilon_{i}=\{0.0001,0.01,0.1\}$ for $i=\{0,1,2\}$.

310

311 Visual saliency refers to the physical, bottom-up distinctness of image details (Fecteau \& Munoz

312 2006). It is a relative property that depends on the degree to which a detail is visually distinct

313 from its background (Wertheim 2010). Since saliency quantifies the relative visual importance of

314 image details saliency maps are frequently used in the weighted recombination phase of multi-

315 scale image fusion schemes (Bavirisetti \& Dhuli 2016b; Cui et al. 2015; Gan et al. 2015).

316 Frequency tuned filtering computes bottom-up saliency as local multi-scale luminance contrast

317 (Achanta et al. 2009). The saliency map $S$ for an image $\boldsymbol{I}$ is computed as

318

319 where

320

321

322

323

324

325

326

327

328

329

330

331

332

$$
S(x, y)=\left\|\boldsymbol{I}_{\mu}-\boldsymbol{I}_{f}(x, y)\right\|
$$

$I_{\mu}$ is the arithmetic mean image feature vector,

$I_{f}$ represents a Gaussian blurred version of the original image, using a $5 \times 5$ separable binomial kernel,

\|\| is the $L_{2}$ norm (Euclidian distance), and

$x, y$ are the pixel coordinates.

A recent and extensive evaluation study comparing 13 state-of-the-art saliency models found that the output of this simple saliency model correlates more strongly with human visual perception than the output produced by any of the other available models (Toet 2011).

In the proposed fusion scheme we first compute saliency maps $S_{X_{i}}$ and $S_{Y_{i}}$ for the individual source layers $X_{i}$ and $Y_{i}, i \in\{0,1,2\}$. Binary weight maps $B W_{X_{i}}$ and $B W_{Y_{i}}$ are then computed by taking the pixelwise maximum of corresponding saliency maps $S_{X_{i}}$ and $S_{Y_{i}}$ :

$$
B W_{X_{i}}(x, y)= \begin{cases}1 & \text { if } S_{X_{i}}(x, y)>S_{Y_{i}}(x, y) \\ 0 & \text { otherwise }\end{cases}
$$

$$
B W_{Y_{i}}(x, y)= \begin{cases}1 & \text { if } S_{Y_{i}}(x, y)>S_{X_{i}}(x, y) \\ 0 & \text { otherwise }\end{cases}
$$

333

334

335

336

337

338

339

340

341

342

The resulting binary weight maps are noisy and typically not well aligned with object boundaries, which may give rise to artefacts in the final fused image. Spatial consistency is therefore restored through guided filtering (GF) of these binary weight maps with the corresponding source layers as guidance images:

$$
W_{X_{i}}=\mathrm{GF}\left(B W_{X_{i}}, X_{i}\right)
$$

$$
W_{Y_{i}}=G F\left(B W_{Y_{i}}, Y_{i}\right)
$$

As noted before guided filtering combines noise reduction with edge preservation, while the output is locally approximately a scaled version of the guidance image. In the present scheme these properties are used to transform the binary weight maps into smooth continuous weight maps through guided filtering with the corresponding source images as guidance images. Figure 
3434 illustrates the process of computing smoothed weight maps by guided filtering of the binary 344 weight maps resulting from the pointwise maximum of the corresponding source layer saliency 345 maps for the tank scene.

346

347

348

350 351

353

354

355

356

357

358

359

360

361

362

363

364

365

366

367

368

369

370

371

372

373

374

375

376

377

378

379

380

381

382

Fused residual layers are then computed as the normalized weighted mean of the corresponding source residual layers:

$$
d F_{i}=\frac{W_{X_{i}} \cdot d X_{i}+W_{Y_{i}} \cdot d Y_{i}}{W_{X_{i}}+W_{Y_{i}}}
$$

The fused image $F$ is finally obtained by adding the fused residual layers to the average value of the coarsest source layers:

$$
F=\frac{X_{3}+Y_{3}}{2}+\sum_{i=0}^{2} d F_{i}
$$

By using guided filtering both in the decomposition stage and in the recombination stage, this proposed fusion scheme optimally benefits from both the multi-scale edge-preserving characteristics (in the iterative framework) and the structure restoring capabilities (through guidance by the original source images) of the guided filter. The method is easy to implement and computationally efficient.

\section{Methods and material}

This section presents the test imagery and computational metrics used to assess the performance of the proposed images fusion scheme in comparison to existing multi-scale fusion schemes.

\subsection{Test imagery}

Figure 2 shows the intensified visual (II), thermal infrared (IR) or near infrared (NIR: scene 12) source images together with the result of the proposed fusion scheme (F) for each of the 12 scenes used in this study. The 12 scenes are part of the TNO Image Fusion Dataset (Toet 2014) with the following identifiers: airplane_in_trees, Barbed_wire_2, Jeep, Kaptein_1123, Marne_07, Marne_11, Marne_15, Reek, tank, Nato_camp_sequence, soldier_bēhind_smoke, Vlasakkers.

\subsection{Multi-scale fusion schemes used for comparison}

In this study we compare the performance of our image fusion scheme with seven other popular image fusion methods based on multi-scale decomposition including the Laplacian pyramid (Burt \& Adelson 1983), the Ratio of Low-Pass pyramid (Toet 1989b), the contrast pyramid (Toet et al. 1989), the filter-subtract-decimate Laplacian pyramid (Burt 1988; Burt \& Kolczynski 1993), the gradient pyramid (Burt 1992; Burt \& Kolczynski 1993), the morphological pyramid ((Toet 1989a)), the discrete wavelet transform (Lemeshewsky 1999; Li et al. 1995; Li et al. 2002; Scheunders \& De Backer 2001), and a shift invariant extension of the discrete wavelet transform (Lemeshewsky 1999; Rockinger 1997; Rockinger 1999; Rockinger \& Fechner 1998). We used 
383 Rockinger's freely available Matlab image fusion toolbox (www.metapix.de/toolbox.htm) to

384

385

386

387

388

389

390

391

392

393

394

395

396

397

398

399

400

401

402

403

404

405

406

407

408

409

410

411

412

413

414

415

416

417

418

419

420 compute these fusion schemes. To allow a straightforward comparison, the number of scale levels is set to 4 in all methods, and simple averaging is used to compute the approximation of the fused image representation at the coarsest spatial scale. Figures 5-9 show the results of the proposed method together with the results of other seven fusion schemes for some of the scenes used in this study (scenes 2-5 and 10).

\subsection{Objective evaluation metrics}

Image fusion results can be evaluated using either subjective or objective measures. Subjective methods are based on psycho-visual testing and are typically expensive in terms of time, effort, and equipment required. Also, in most cases, there is only little difference among fusion results. This makes it difficult to subjectively perform the evaluation of fusion results. Therefore, many objective evaluation methods have been developed (for an overview see e.g. (Li et al. 2010; Liu et al. 2012). However, so far, there is no universally accepted metric to objectively evaluate the image fusion results. In this paper, we use four frequently applied computational metrics to objectively evaluate and compare the performance of different image fusion methods. The metrics we use are Entropy, the Mean Structural Similarity Index (MSSIM), Normalized Mutual Information (NMI), and Normalized Feature Mutual Information (NFMI). These metrics will be briefly discussed in the following sections.

\subsubsection{Entropy}

Entropy (E) is a measure of the information content in a fused image F. Entropy is defined as

$$
E_{F}=-\sum_{i=0}^{L-1} P_{F}(i) \log P_{F}(i)
$$

where $P_{F}(i)$ indicates the probability that a pixel in the fused image $\mathrm{F}$ has a gray value $i$, and the gray values range from 0 to $L$. The larger the entropy is, the more informative the fused image is. A fused image is more informative than either of its source images when its entropy is higher than the entropy of its source images.

\subsubsection{Mean Structural Similarity Index}

The Structural Similarity (SSIM: (Wang et al. 2004)) index is a stabilized version of the Universal Image Quality Index (UIQ: (Wang \& Bovik 2002)) which can be used to quantify the structural similarity between a source image $\mathrm{A}$ and a fused image $\mathrm{F}$ :

$$
\operatorname{SSIM}_{x, y}=\frac{2 \mu_{x} \mu_{y}+C_{1}}{\mu_{x}{ }^{2}+\mu_{y}{ }^{2}+C_{1}} \cdot \frac{2 \sigma_{x} \sigma_{y}+C_{2}}{\sigma_{x}^{2}+\sigma_{y}^{2}+C_{2}} \cdot \frac{\sigma_{x y}+C_{3}}{\sigma_{x} \sigma_{y}+C_{3}} .
$$

where $x$ and $y$ represent local windows of size $M \times N$ in respectively $A$ and $F$, and 
421

422

423

424

425

426

427

428

429

430

431

432

433

434

435

436

437

438

439

440

441

442

443

444

445

446

447

448

449

450

451

452

453

454

$$
\begin{gathered}
\mu_{x}=\frac{1}{M \times N} \sum_{i=1}^{M} \sum_{j=1}^{N} x(i, j) \quad, \quad \mu_{y}=\frac{1}{M \times N} \sum_{i=1}^{M} \sum_{j=1}^{N} y(i, j) \\
\sigma_{x}^{2}=\frac{1}{M \times N} \sum_{i=1}^{M} \sum_{j=1}^{N}\left(x(i, j)-\mu_{x}\right)^{2} \quad, \quad \sigma_{y}^{2}=\frac{1}{M \times N} \sum_{i=1}^{M} \sum_{j=1}^{N}\left(y(i, j)-\mu_{y}\right)^{2} \\
\sigma_{x y}^{2}=\frac{1}{M \times N} \sum_{i=1}^{M} \sum_{j=1}^{N}\left(x(i, j)-\mu_{x}\right)\left(y(i, j)-\mu_{y}\right)
\end{gathered}
$$

By default, the stabilizing constants are set to $C_{1}=(0.01 \cdot L)^{2}, C_{2}=(0.03 \cdot L)^{2}$ and $C_{3}=C_{2} / 2$, where $\mathrm{L}$ is the maximal gray value. The value of SSIM is bounded and ranges between -1 and 1 (it is 1 only when both images are identical). The SSIM is typically computed over a sliding window to compare local patterns of pixel intensities that have been normalized for luminance and contrast. The Mean Structural Similarity (MSSIM) index quantifies the overall similarity between a source image $\mathrm{A}$ and a fused image $\mathrm{F}$ :

$$
\operatorname{MSSIM}_{A, F}=\frac{1}{N_{w}} \sum_{i=1}^{N_{w}} \operatorname{SSIM}_{x_{i}, y_{i}}
$$

where $N_{w}$ represents the number of local windows of the image. An overall image fusion quality index can then be defined as the mean MSSIM values between each of the source images and the fused result:

$$
\operatorname{MSSIM}_{F}^{A, B}=\frac{\operatorname{MSSIM}_{A, F}+\operatorname{MSSIM}_{B, F}}{2}
$$

$\operatorname{MSSIM}_{F}^{A, B}$ ranges between -1 and 1 (it is 1 only when both images are identical).

\subsubsection{Normalized Mutual Information}

Mutual Information (MI) measures the amount of information that two images have in common. It can be used to quantify the amount of information from a source image that is transferred to a fused image (Qu et al. 2002). The mutual information $M I_{A F}$ between a source image $\mathrm{A}$ and a fused image $\mathrm{F}$ is defined as:

$$
M I_{A, F}=\sum_{i, j} P_{A, F}(i, j) \log \frac{P_{A, F}(i, j)}{P_{A}(i) P_{F}(j)}
$$

where $P_{A}(i)$ and $P_{F}(j)$ are the probability density functions in the individual images, and $P_{A F}(i, j)$ is the joint probability density function.

The traditional mutual information metric is unstable and may bias the measure towards the source image with the highest entropy. This problem can be resolved by computing the normalized mutual information (NMI) as follows (Hossny et al. 2008):

$$
N M I_{F}^{A, B}=\frac{M I_{A, F}}{H_{A}+H_{F}}+\frac{M I_{B, F}}{H_{B}+H_{F}}
$$

where $H_{A}, H_{B}$ and $H_{F}$ are the marginal entropy of $\mathrm{A}, \mathrm{B}$ and $\mathrm{F}$, and $M I_{A, F}$ and $M I_{B, F}$ represent the mutual information between respectively the source image $\mathrm{A}$ and the fused image $\mathrm{F}$ and between the source image B and the fused image F. A higher value of NMI indicates that more information from the source images is transferred to the fused image. The NMI metric varies between 0 and 1 . 


\subsubsection{Normalized Feature Mutual Information}

457

458

459

460

461

462

463

464

465

466

467

468

469

470

471

472

473

474

475

476

477

478

479

480

481

482

483

484

485

486

487

488

489

490

491

492

493

494

The Feature Mutual Information (FMI) metric calculates the amount of image features that two images have in common (Haghighat \& Razian 2014; Haghighat et al. 2011). This method outperforms other metrics (e.g., E, NMI) in consistency with the subjective quality measures. Previously proposed MI-based image fusion quality metrics use the image histograms to compute the amount of information a source and fused image have in common (Cvejic et al. 2006; Qu et al. 2002). However, image histograms contain no information about local image structure (spatial features or local image quality) and only provide statistical measures of the number of pixels in a specific gray-level. However, since meaningful image information is contained in visual features, image fusion quality measures should measure the extent to which these visual features are transferred into the fused image from each of the source images. The Feature Mutual Information (FMI) metric calculates the mutual information between image feature maps (Haghighat \& Razian 2014; Haghighat et al. 2011). A typical image feature map is for instance the gradient map, which contains information about the pixel neighborhoods, edge strength and directions, texture and contrast. Given two source images as A and B and their fused image as F, the FMI metric first extracts feature maps of the source and fused images using a feature extraction method (e.g., gradient). After feature extraction, the feature images $A^{\prime}, B^{\prime}$ and $F^{\prime}$ are normalized to create their marginal probability density functions $P_{A^{\prime}}, P_{B^{\prime}}$ and $P_{F^{\prime}}$. The joint probability density functions $P_{A^{\prime}, F^{\prime}}$ and $P_{B^{\prime}, F^{\prime}}$ are then estimated from the marginal distributions using Nelsen's method (Nelsen 1987). The algorithm is described in more detail elsewhere (Haghighat et al. 2011). The FMI metric between a source image A and a fused image $\mathrm{F}$ is then given by

$$
F M I_{A, F}=M I_{A^{\prime}, F^{\prime}}=\sum_{i, j} P_{A^{\prime}, F^{\prime}}(i, j) \log \frac{P_{A^{\prime}, F^{\prime}}(i, j)}{P_{A^{\prime}}(i) P_{F^{\prime}}(j)}
$$

and the normalized feature mutual information (FMI) can be computed as follows

$$
F M I_{F}^{A, B}=\frac{M I_{A^{\prime}, F^{\prime}}}{H_{A^{\prime}}+H_{F^{\prime}}}+\frac{M I_{B^{\prime}, F^{\prime}}}{H_{B^{\prime}}+H_{F^{\prime}}}
$$

In practice the FMI is computed locally over small corresponding windows between the source and the fused images and averaged over all windows covering the image plane (Haghighat \& Razian 2014).

\section{Results}

\subsection{Fusion evaluation}

Here we assess the performance of the proposed image fusion scheme on the intensified visual and thermal infrared images for each of the 12 selected scenes, using Entropy, the Mean Structural Similarity Index (MSSIM), Normalized Mutual Information (NMI), and Normalized Feature Mutual Information (NFMI) as the objective performance measures. We also compare the results of the proposed method with those of seven other popular multi-scale fusion schemes. 
495

496

497

498

499

500

501

502

503

504

505

506

507

508

509

510

511

512

513

514

515

516

517

518

519

520

521

522

523

524

525

526

527

528

529

530

531

532

533

534

535

536

537

538

Table 1 lists the entropy of the fused result for the proposed method (IGF) and all seven multiscale comparison methods (Contrast Pyramid, DWT, Gradient Pyramid, Laplace Pyramid, Morphological Pyramid, Ratio Pyramid, SIDWT). It appears that IGF produces a fused image with the highest entropy for 9 of the 12 test scenes. Note that a larger entropy implies more edge information, but it does not mean that the additional edges are indeed meaningful (they may result from over enhancement or noise). Therefore, we also need to consider structural information metrics.

Table 2 shows that IGF outperforms all other multi-scale methods tested here in terms of MSSIM. This means that the mean overall structural similarity between both source images the fused image $\mathrm{F}$ is largest for the proposed method.

Table 3 shows that IGF also outperforms all other multi-scale methods tested here in terms of NMI. This indicates that the proposed IGF fusion scheme transfers more information from the source images to the fused image than any of the other methods.

Table 4 shows that IGF also outperforms 10 of the 12 other multi-scale methods tested here in terms of NFMI. IGF is only outperformed by SIDWT for scene 1 and by the Contrast Pyramid for scene 7. This implies that fused images produced by the proposed IGF scheme typically have a larger amount of image features in common with their source images than the results of most other fusion schemes.

Summarizing, the proposed IGF fusion scheme appears to outperform the other multi-scale fusion methods investigated here in most of the conditions tested.

\subsection{Runtime}

In this study we used a Matlab implementation of the GF and IGF written by Zhang et al. (2014) that is freely available from the authors (at http://www.cs.cuhk.edu.hk/ leojia/projects/rollguidance). We made no effort to optimize the code of the algorithms. We conducted a runtime test on a Dell Latitude laptop with an Intel i5 2 GHz CPU and 8 GB memory. The algorithms were implemented in Matlab 2016a. Only a single thread was used without involving any SIMD instructions. For this test we used the set of 12 test images described in Section 5.1. As noted before, the filter size and regularization parameters used in this study are respectively set to $r_{i}=\{5,10,30\}$ and $\varepsilon_{i}=\{0.0001,0.01,0.1\}$ for spatial scale levels $i=\{0,1,2\}$. The mean runtime of the proposed fusion method was $0.61 \pm 0.05$ seconds.

\section{Discussion and conclusions}

We propose a multi-scale image fusion scheme based on guided filtering. Iterative guided filtering is used to decompose the source images into approximation and residual layers. Initial binary weighting maps are computed as the pixelwise maximum of the individual source saliency maps, obtained from frequency tuned filtering. Spatially consistent and smooth 
539 weighting maps are then obtained through guided filtering of the binary weighting maps with 540 their corresponding source layers as guidance images. Saliency weighted recombination of the 541 individual source residual layers and the mean of the coarsest scale source layers finally yields 542 the fused image. The proposed multi-scale image fusion scheme achieves spatial consistency by 543 using guided filtering both at the decomposition and at the recombination stage of the multi-scale 544 fusion process. Application to multiband visual (intensified) and thermal infrared imagery 545 demonstrates that the proposed method obtains state-of-the-art performance for the fusion of 546 multispectral nightvision images. The method has a simple implementation and is 547 computationally efficient. 
548

549

550

551

552

553

554

555

556

557

558

559

560

561

562

563

564

565

566

567

568

569

570

571

572

573

574

575

576

577

578

579

580

581

582

583

584

585

586

587

588

589

590

591

592

593

\section{References}

Achanta R, Hemami S, Estrada F, and Süsstrunk S. 2009. Frequency-tuned salient region detection. In: Hemami S, Estrada F, and Susstrunk S, eds. IEEE International Conference on Computer Vision and Pattern Recognition (CVPR 2009): IEEE Press, 1597-1604. DOI: $10.1109 /$ CVPR.2009.5206596.

Agarwal J, and Bedi SS. 2015. Implementation of hybrid image fusion technique for feature enhancement in medical diagnosis. Human-centric Computing and Information Sciences 5(1):1-17. DOI: 10.1186/s13673-014-0020-z.

Bavirisetti DP, and Dhuli R. 2016a. Fusion of infrared and visible sensor images based on anisotropic diffusion and Karhunen-Loeve transform. IEEE Sensors Journal 16(1):203209. DOI: 10.1109/JSEN.2015.2478655.

Bavirisetti DP, and Dhuli R. 2016b. Two-scale image fusion of visible and infrared images using saliency detection. Infrared Physics \& Technology 76:52-64. DOI: 10.1016/j.infrared.2016.01.009.

Beyan C, Yigit A, and Temizel A. 2011. Fusion of thermal- and visible-band video for abandoned object detection. Journal of Electronic Imaging 20(033001):1-12. DOI: $10.1117 / 1.3602204$.

Bhatnagar G, and Wu QMJ. 2011. Human visual system based framework for concealed weapon detection. The 2011 Canadian Conference on Computer and Robot Vision (CRV): IEEE, 250-256. DOI: 10.1109/CRV.2011.40.

Biswas B, Chakrabarti A, and Dey KN. 2015. Spine medical image fusion using wiener filter in shearlet domain. IEEE 2nd International Conference on Recent Trends in Information Systems (ReTIS 2015): IEEE, 387-392. DOI: 10.1109/ReTIS.2015.7232910.

Black MJ, Sapiro G, Marimont DH, and Heeger D. 1998. Robust anisotropic diffusion. IEEE Transactions on Image Processing 7(3):421-432. DOI: 10.1109/83.661192.

Blum RS, and Liu Z. 2006. Multi-sensor image fusion and its applications. Boca Raton, Florida, USA: CRC Press, Taylor \& Francis Group.

Bulanona DM, Burks TF, and Alchanatis V. 2009. Image fusion of visible and thermal images for fruit detection. Biosystems Engineering 103(1):12-22. DOI: 10.1016/j.biosystemseng.2009.02.009.

Burt PJ. 1988. Smart sensing with a pyramid vision machine. Proceedings IEEE 76(8):10061015. DOI: $10.1109 / 5.5971$.

Burt PJ. 1992. A gradient pyramid basis for pattern-selective image fusion. SID International Symposium 1992. Playa del Rey, CA: Society for Information Display, 467-470.

Burt PJ, and Adelson EH. 1983. The Laplacian pyramid as a compact image code. IEEE Transactions on Communications 31(4):532-540. DOI: 10.1109/TCOM.1983.1095851.

Burt PJ, and Kolczynski RJ. 1993. Enhanced image capture through fusion. Fourth International Conference on Computer Vision. Washington, USA: IEEE Computer Society Press. 173182.

Cui G, Feng H, Xu Z, Li Q, and Chen Y. 2015. Detail preserved fusion of visible and infrared images using regional saliency extraction and multi-scale image decomposition. Optics Communications 341:199-209. DOI: 10.1016/j.optcom.2014.12.032.

Cvejic N, Canagarajah CN, and Bull DR. 2006. Image fusion metric based on mutual information and Tsallis entropy. Electronics Letters 42(11):626-627. DOI: 10.1049/el:20060693. 
594

595

596

597

598

599

600

601

602

603

604

605

606

607

608

609

610

611

612

613

614

615

616

617

618

619

620

621

622

623

624

625

626

627

628

629

630

631

632

633

634

635

636

637

638
Daneshvar S, and Ghassemian H. 2010. MRI and PET image fusion by combining IHS and retina-inspired models. Information Fusion 11(2):114-123. DOI: 10.1016/j.inffus.2009.05.003.

Farbman Z, Fattal R, Lischinski D, and Szeliski R. 2008. Edge-preserving decompositions for multi-scale tone and detail manipulation. ACM Transactions on Graphics 27(3 - Article No. 67):1-10. DOI: 10.1145/1360612.1360666.

Fecteau JH, and Munoz DP. 2006. Salience, relevance, and firing: A priority map for target selection. Trends in Cognitive Sciences 10(8):382-390. DOI: 10.1016/j.tics.2006.06.011.

Gan W, Wu X, Wu W, Yang X, Ren C, He X, and Liu K. 2015. Infrared and visible image fusion with the use of multi-scale edge-preserving decomposition and guided image filter. Infrared Physics \& Technology 72:37-51. DOI: 10.1016/j.infrared.2015.07.003.

Ghassemian H. 2001. A retina based multi-resolution image-fusion. IEEE International Geoscience and Remote Sensing Symposium (IGRSS2001): IEEE, 709-711. DOI: 10.1109/IGARSS.2001.976610.

Haghighat M, and Razian MA. 2014. Fast-FMI: Non-reference image fusion metric. Piscataway, NJ, USA: IEEE. 1-3. DOI: 1109/ICAICT.2014.7036000.

Haghighat MBA, Aghagolzadeh A, and Seyedarabi H. 2011. A non-reference image fusion metric based on mutual information of image features. Computers \& Electrical Engineering 37(5):744-756. DOI: 10.1016/j.compeleceng.2011.07.012.

He K, Sun J, and Tang X. 2013. Guided image filtering. IEEE Transactions on Pattern Analysis and Machine Intelligence 35(6):1397-1409. DOI: 10.1109/TPAMI.2012.213.

Hossny M, Nahavandi S, and Creighton D. 2008. Comments on "Information measure for performance of image fusion". Electronics Letters 44(18):1066-1067. DOI: 10.1049/el:20081754.

Jacobson NP, and Gupta MR. 2005. Design goals and solutions for display of hyperspectral images. IEEE Transactions on Geoscience and Remote Sensing 43(11):2684-2692. DOI: 10.1109/TGRS.2005.857623.

Jacobson NP, Gupta MR, and Cole JB. 2007. Linear fusion of image sets for display. IEEE Transactions on Geoscience and Remote Sensing 45(10):3277-3288. DOI: 10.1109/TGRS.2007.903598.

Jiang D, Zhuang D, Huan Y, and Fu J. 2011. Survey of multispectral image fusion techniques in remote sensing applications. In: Zheng Y, ed. Image Fusion and Its Applications. Rijeka, Croatia: InTech Open, 1-22.

Kong SG, Heo J, Boughorbel F, Zheng Y, Abidi BR, Koschan A, Yi M, and Abidi MA. 2007. Multiscale fusion of visible and thermal IR images for illumination-invariant face recognition. International Journal of Computer Vision 71(2):215-233. DOI: 10.1007/s11263-006-6655-0.

Kong W, Wang B, and Lei Y. 2015. Technique for infrared and visible image fusion based on non-subsampled shearlet transform and spiking cortical model. Infrared Physics \& Technology 71:87-98. DOI: 10.1016/j.infrared.2015.02.008.

Kumar BKS. 2013. Image fusion based on pixel significance using cross bilateral filter. Signal, Image and Video Processing 9(5):1193-1204. DOI: 10.1007/s11760-013-0556-9.

Lemeshewsky GP. 1999. Multispectral multisensor image fusion using wavelet transforms. In: Park SJ, and Juday RD, eds. Bellingham, WA: The International Society for Optical Engineering. 214-222. 
639

640

641

642

643

644

645

646

647

648

649

650

651

652

653

654

655

656

657

658

659

660

661

662

663

664

665

666

667

668

669

670

671

672

673

674

675

676

677

678

679

680

681

682

Lepley JJ, and Averill MT. 2011. Detection of buried mines and explosive objects using dualband thermal imagery. In: Harmon RS, Holloway JH, and Broach JT, eds. Detection and Sensing of Mines, Explosive Objects, and Obscured Targets XVI, vol. SPIE-8017. Bellingham, WA, USA: The International Society for Optical Engineering, 80171V80171-80112. DOI: 10.1117/12.883375.

Li H, Manjunath BS, and Mitra SK. 1995. Multisensor image fusion using the wavelet transform. Computer Vision, Graphics and Image Processing: Graphical Models and Image Processing 57(3):235-245.

Li S, Kang X, and Hu J. 2013. Image fusion with guided filtering. IEEE Transactions on Image Processing 22(7):2864-2875. DOI: 10.1109/TIP.2013.2244222.

Li S, Kwok JT, and Wang Y. 2002. Using the discrete wavelet frame transform to merge Landsat TM and SPOT panchromatic images. Information Fusion 3(1):17-23.

Li S, Li Z, and Gong J. 2010. Multivariate statistical analysis of measures for assessing the quality of image fusion. International Journal of Image and Data Fusion 1(1):47-66. DOI: $10.1080 / 19479830903562009$.

Liu X, Mei W, Du H, and Bei J. 2016. A novel image fusion algorithm based on nonsubsampled shearlet transform and morphological component analysis. Signal, Image and Video Processing 10(5):959-966. DOI: 10.1007/s11760-015-0846-5.

Liu Z, Blasch EP, Xue Z, Zhao J, Laganière R, and Wu W. 2012. Objective assessment of multiresolution image fusion algorithms for context enhancement in night vision: A comparative study. IEEE Transactions on Pattern Analysis and Machine Intelligence 34(1):94-109. DOI: doi.ieeecomputersociety.org/10.1109/TPAMI.2011.109.

Liu Z, Xue Z, Blum RS, and Laganiëre R. 2006. Concealed weapon detection and visualization in a synthesized image. Pattern Analysis \& Applications 8(4):375-389. DOI: 10.1007/s10044-005-0020-8.

Motamed C, Lherbier R, and Hamad D. 2005. A multi-sensor validation approach for human activity monitoring. 7th International Conference on Information Fusion (Information Fusion 2005): IEEE. DOI: 10.1109/ICIF.2005.1592039.

Nelsen RB. 1987. Discrete bivariate distributions with given marginals and correlation. Communications in Statistics - Simulation and Computation 16(1):199-208. DOI: 10.1080/03610918708812585.

O'Brien MA, and Irvine JM. 2004. Information fusion for feature extraction and the development of geospatial information. 7th International Conference on Information Fusion: ISIF, 976-982.

Perona P, and Malik J. 1990. Scale-space and edge detection using anisotropic diffusion. IEEE Transactions on Pattern Analysis and Machine Intelligence 12(7):629-639. DOI: 10.1109/34.56205.

Petrovic VS, and Xydeas CS. 2003. Sensor noise effects on signal-level image fusion performance. Information Fusion 4(3):167-183. DOI: 10.1016/S1566-2535(03)00035-6.

Petschnigg G, Agrawala M, Hoppe H, Szeliski R, Cohen M, and Toyama K. 2004. Digital photography with flash and no-flash image pairs. New York, USA: ACM Press. 664-672. DOI: $10.1145 / 1015706.1015777$.

Qu GH, Zhang DL, and Yan PF. 2002. Information measure for performance of image fusion. Electronics Letters 38(7):313-315. DOI: 10.1049/el:20020212. 
683 Rockinger O. 1997. Image sequence fusion using a shift-invariant wavelet transform. IEEE

684

685

686

687

688

689

690

691

692

693

694

695

696

697

698

699

700

701

702

703

704

705

706

707

708

709

710

711

712

713

714

715

716

717

718

719

720

721

722

723

724

725

726

727
International Conference on Image Processing, vol. III: IEEE, 288-291. DOI: 10.1109/ICIP.1997.632093.

Rockinger O. 1999. Multiresolution-Verfahren zur Fusion dynamischer Bildfolge Ph.D. Thesis. Technische Universität Berlin.

Rockinger O, and Fechner T. 1998. Pixel-level image fusion: the case of image sequences. In: Kadar I, ed. Signal Processing, Sensor Fusion, and Target Recognition VII, vol. SPIE3374: The International Society for Optical Engineering, 378-388. DOI: $10.1117 / 12.327135$.

Scheunders P, and De Backer S. 2001. Fusion and merging of multispectral images using multiscale fundamental forms. Journal of the Optical Society of America A 18(10):24682477. DOI: $10.1364 /$ JOSAA.18.002468.

Shah P, Reddy BCS, Merchant S, and Desai U. 2013. Context enhancement to reveal a camouflaged target and to assist target localization by fusion of multispectral surveillance videos. Signal, Image and Video Processing 7(3):537-552. DOI: 10.1007/s11760-0110257-1.

Singh R, and Khare A. 2014. Fusion of multimodal medical images using Daubechies complex wavelet transform - A multiresolution approach. Information Fusion 19:49-60. DOI: 10.1016/j.inffus.2012.09.005.

Singh R, Vatsa M, and Noore A. 2008. Integrated multilevel image fusion and match score fusion of visible and infrared face images for robust face recognition. Pattern Recognition 41(3):880-893. DOI: 10.1016/j.patcog.2007.06.022.

Tao C, Junping Z, and Ye Z. 2005. Remote sensing image fusion based on ridgelet transform. 2005 IEEE International Geoscience and Remote Sensing Symposium (IGARSS '05), vol. 2: IEEE, 1150-1153. DOI: 10.1109/IGARSS.2005.1525320.

Tian YP, Zhou KY, Feng X, Yu SL, Liang H, and Liang B. 2009. Image fusion for infrared thermography and inspection of pressure vessel. Journal of Pressure Vessel Technology 131(2 - article no. 021502):1-5. DOI: 10.1115/1.3066801.

Toet A. 1989a. A morphological pyramidal image decomposition. Pattern Recognition Letters 9(4):255-261. DOI: 10.1016/0167-8655(89)90004-4.

Toet A. 1989b. Image fusion by a ratio of low-pass pyramid. Pattern Recognition Letters 9(4):245-253. DOI: 10.1016/0167-8655(89)90003-2.

Toet A. 2003. Color image fusion for concealed weapon detection. In: Carapezza EM, ed. Sensors, and command, control, communications, and intelligence (C3I) technologies for homeland defense and law enforcement II, vol. SPIE-5071. Bellingham, WA., USA: SPIE, 372-379. DOI: 10.1117/12.484845.

Toet A. 2011. Computational versus psychophysical image saliency: a comparative evaluation study. IEEE Transactions on Pattern Analysis and Machine Intelligence 33(11):21312146. DOI: 10.1109/TPAMI.2011.53.

Toet A. 2014. TNO Image fusion dataset. DOI: $10.6084 / \mathrm{m} 9$.figshare. 1008029 .

Toet A, IJspeert JK, Waxman AM, and Aguilar M. 1997. Fusion of visible and thermal imagery improves situational awareness. Displays 18(2):85-95. DOI: 10.1016/S01419382(97)00014-0.

Toet A, van Ruyven LJ, and Valeton JM. 1989. Merging thermal and visual images by a contrast pyramid. Optical Engineering 28(7):789-792. DOI: 10.1117/12.7977034. 
728

729

730

731

732

733

734

735

736

737

738

739

740

741

742

743

744

745

746

747

748

749

750

751

752

753

754

755

756

757

758

759

760

761

762

763

764

765

766

767

768

769

770

771

772

773
Tomasi C, and Manduchi R. 1998. Bilateral filtering for gray and color images. IEEE Sixth International Conference on Computer Vision: IEEE. 839-846. DOI: 10.1109/ICCV.1998.710815.

Wang L, Li B, and Tian Lf. 2014. Multi-modal medical image fusion using the inter-scale and intra-scale dependencies between image shift-invariant shearlet coefficients. Information Fusion 19:20-28. DOI: 10.1016/j.inffus.2012.03.002.

Wang Z, and Bovik AC. 2002. A universal image quality index. IEEE Signal Processing Letters 9(3):81-84.

Wang Z, Bovik AC, Sheikh HR, and Simoncelli EP. 2004. Image quality assessment: from error visibility to structural similarity. IEEE Transactions on Image Processing 13(4):600-612.

Wertheim AH. 2010. Visual conspicuity: A new simple standard, its reliability, validity and applicability. Ergonomics 53(3):421-442. DOI: 10.1080/00140130903483705.

Xue Z, and Blum RS. 2003. Concealed weapon detection using color image fusion. Sixth International Conference on Information Fusion (FUSION 2003): IEEE, 622-627. DOI: 10.1109/ICIF.2003.177504.

Xue Z, Blum RS, and Li Y. 2002. Fusion of visual and IR images for concealed weapon detection. Fifth International Conference on Information Fusion, vol. 2: IEEE, 11981205. DOI: $10.1109 /$ ICIF.2002.1020949.

Yajie W, and Mowu L. 2009. Image fusion based concealed weapon detection. International Conference on Computational Intelligence and Software Engineering 2009 (CiSE 2009): IEEE, 1-4. DOI: 10.1109/CISE.2009.5364581.

Yang S, Wang M, Jiao L, Wu R, and Wang Z. 2010. Image fusion based on a new contourlet packet. Information Fusion 11(2):78-84. DOI: 10.1016/j.inffus.2009.05.001.

Yang W, and Liu J-R. 2013. Research and development of medical image fusion. 2013 IEEE International Conference on Medical Imaging Physics and Engineering (ICMIPE): IEEE, 307-309. DOI: 10.1109/ICMIPE.2013.6864557.

Yong J, and Minghui W. 2014. Image fusion using multiscale edge-preserving decomposition based on weighted least squares filter. IET Image Processing 8(3):183-190. DOI: 10.1049/iet-ipr.2013.0429.

Zhang B, Lu X, Pei H, and Zhao Y. 2015. A fusion algorithm for infrared and visible images based on saliency analysis and non-subsampled Shearlet transform. Infrared Physics \& Technology 73:286-297. DOI: 10.1016/j.infrared.2015.10.004.

Zhang Q, Shen X, Xu L, and Jia J. 2014. Rolling guidance filter. In: Fleet D, Pajdla T, Schiele B, and Tuytelaars T, eds. 13th European Conference on Computer Vision (ECCV 2014), vol. III: Springer International Publishing, 815-830. DOI: 10.1007/978-3-319-10578-9_53.

Zhao J, Feng H, Xu Z, Li Q, and Liu T. 2013. Detail enhanced multi-source fusion using visual weight map extraction based on multi scale edge preserving decomposition. Optics Communications 287:45-52. DOI: 10.1016/j.optcom.2012.08.070.

Zhu Z, and Huang TS. 2007. Multimodal surveillance: Sensors, algorithms and systems. Norwood, MA, USA: Artech House Publishers.

Zitová B, Beneš M, and Blažek J. 2011. Image fusion for art analysis. Computer Vision and Image Analysis of Art II, vol. SPIE-7869. Bellingham, WA, USA: The International Society for Optical Engineering, 7869081-7869089. DOI: 10.1117/12.872420.

Zou X, and Bhanu B. 2005. Tracking humans using multi-modal fusion. 2nd Joint IEEE International Workshop on Object Tracking and Classification in and Beyond the Visible Spectrum (OTCBVS'05): IEEE, W01-30-01-08. DOI: 10.1109/CVPR.2005.545. 
775

776

Figures

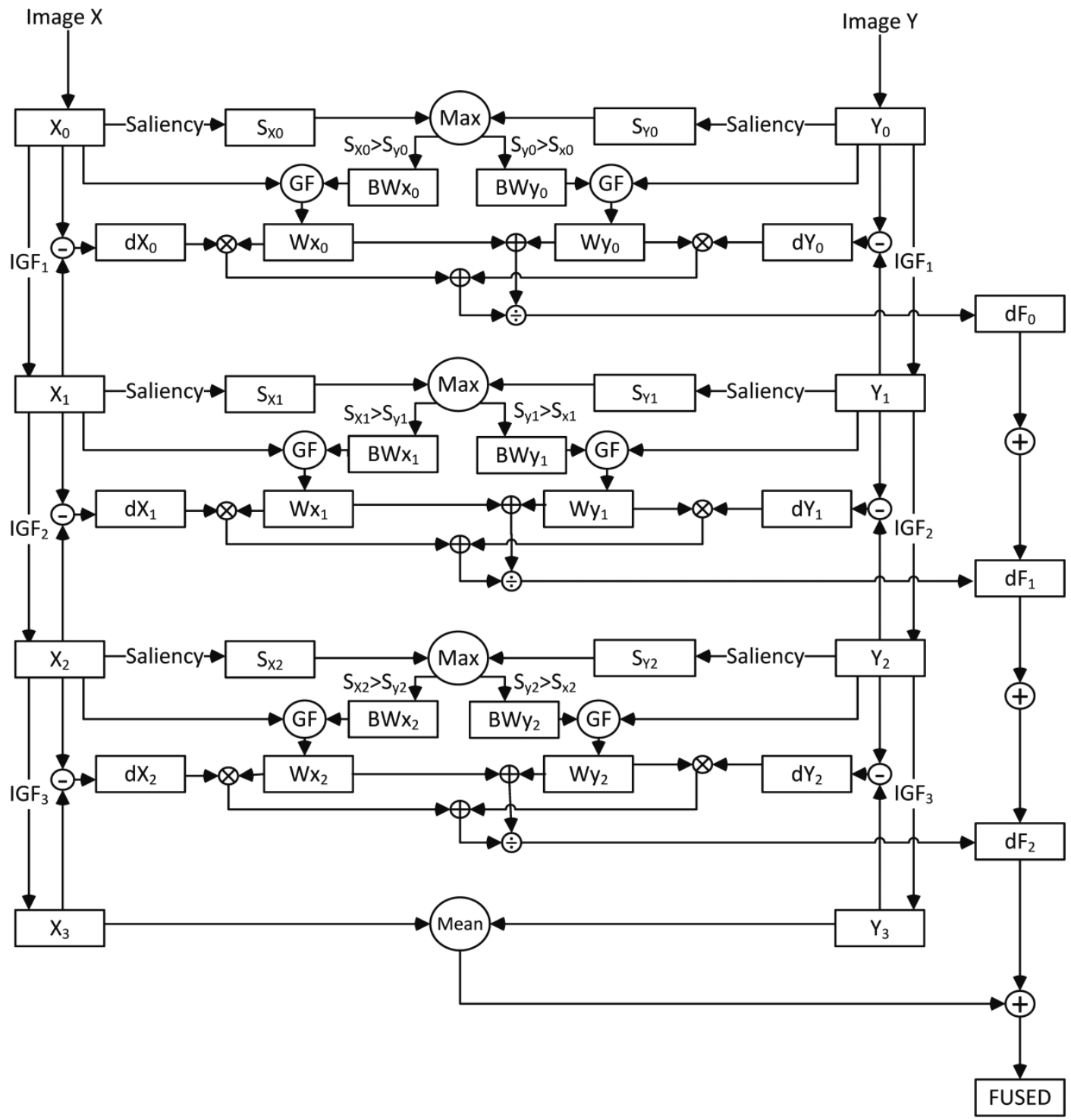

777

778

779

780

781

782

783

784

785

786

787

\section{Figure 1. Flow chart of the proposed image fusion scheme.}

The processing scheme is illustrated for two source images $\mathrm{X}$ and $\mathrm{Y}$ and 4 resolution levels (03). $X_{0}$ and $Y_{0}$ are the original input images, while $X_{i}$ and $Y_{i}$ represent successively lower resolution versions obtained by iterative guided filtering. 'Saliency' represents the frequencytuned saliency transformation, 'Max' and 'Mean' respectively denote the pointwise maximum and mean operators, '(I)GF' means (Iterative) Guided Filtering, ' $d X$ ', ' $d Y$ ' and ' $d F$ ' are respectively the original and fused detail layers, ' $\mathrm{BW}$ ' the binary weight maps, and ' $\mathrm{W}$ ' the smooth weight maps. 

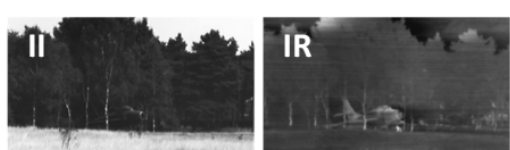

Scene 1
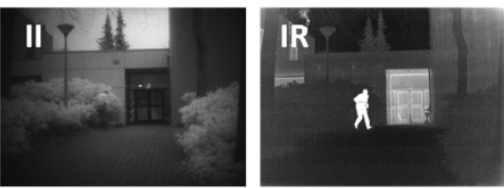

Scene 3
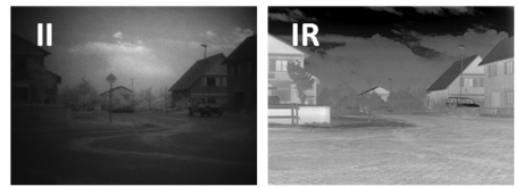

Scene 5
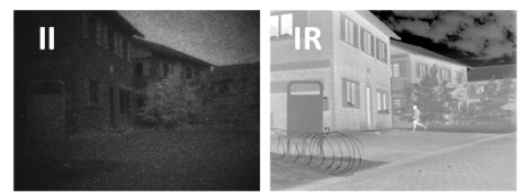

Scene 7
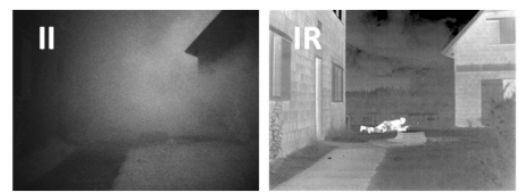

Scene 9

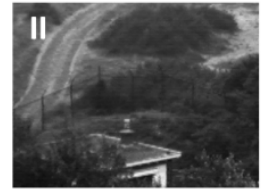

788

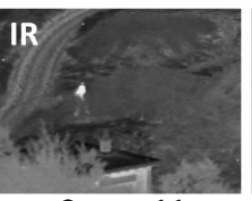

Scene 11
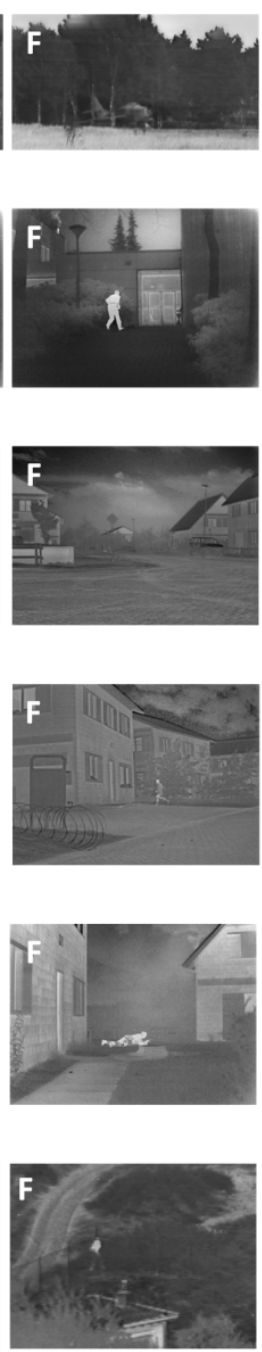

789

790

791

792

793

794

795

796 study.
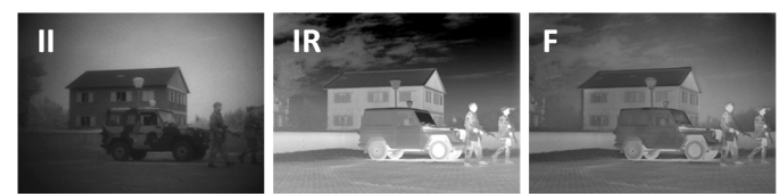

Scene 2
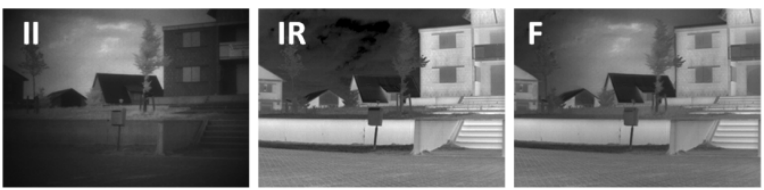

Scene 4
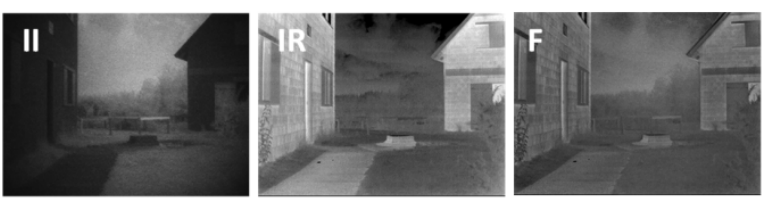

Scene 6
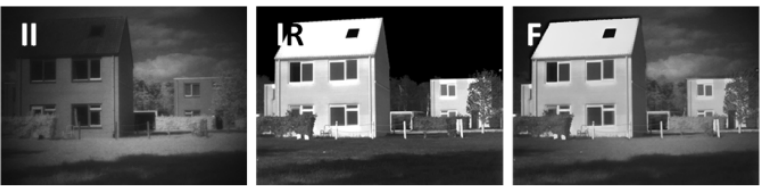

Scene 8
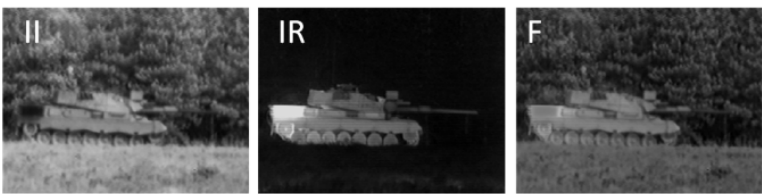

Scene 10
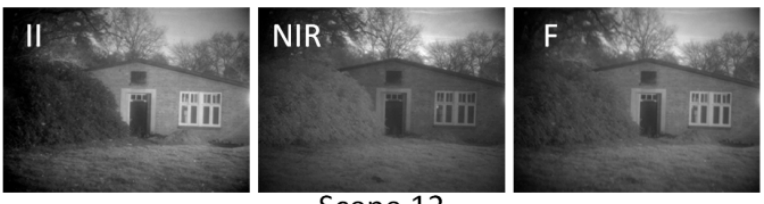

Scene 12

Figure 2. Original input and fused images for all 12 scenes.

The intensified visual (II), thermal infrared (IR) or near infrared (NIR: scene 12) source images together with the result of the proposed fusion scheme $(\mathrm{F})$ for each of the 12 scenes used in this 
797
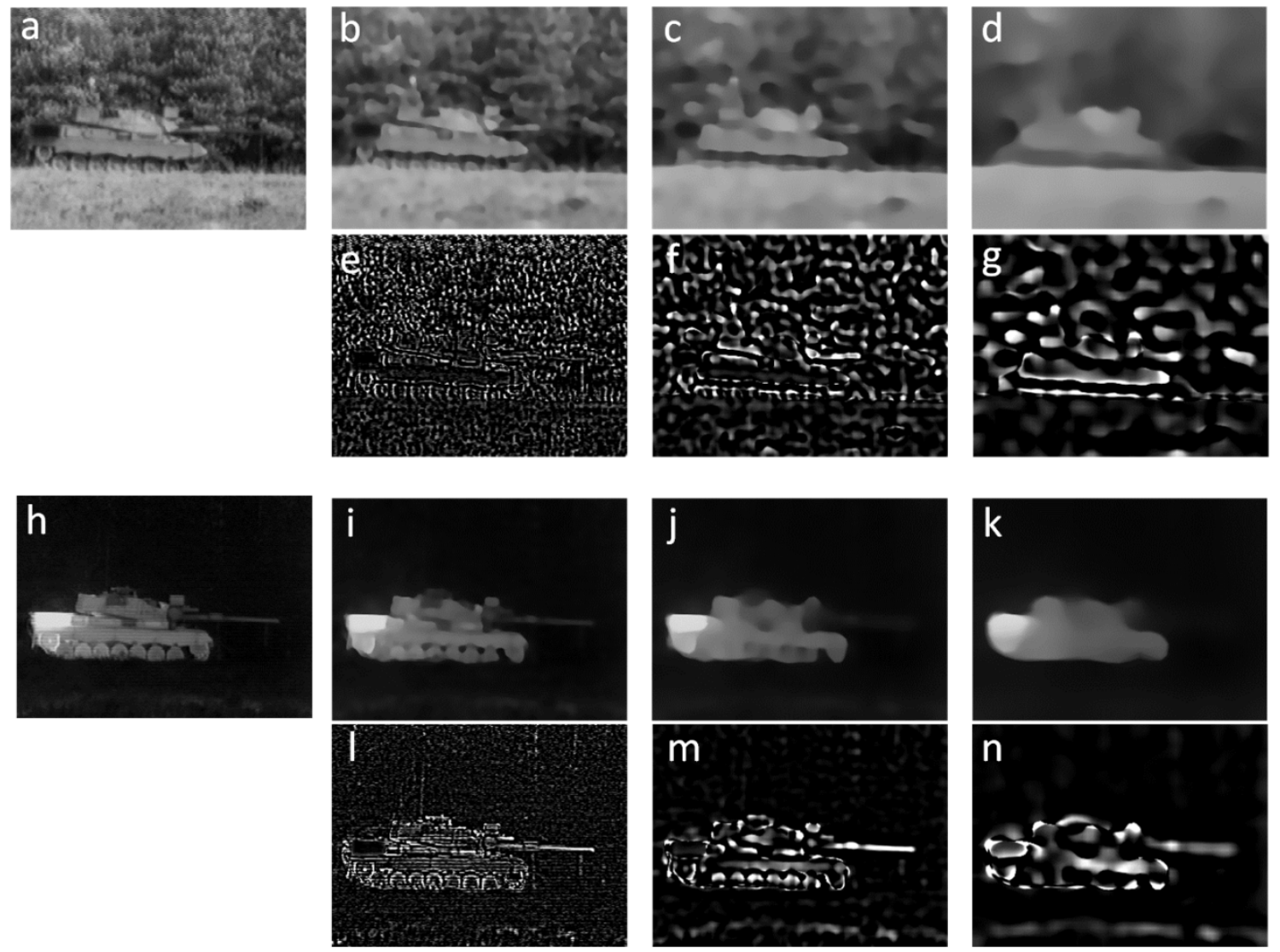

799

800 Figure 3. Base and detail layers for the tank scene.

801 Original intensified visual (a) and thermal infrared (h) images for scene nr. 10, with their

802 respective base (b-d and i-k) and detail (e-g and l-n) layers at successively lower levels of 803 resolution.

804

805 
806
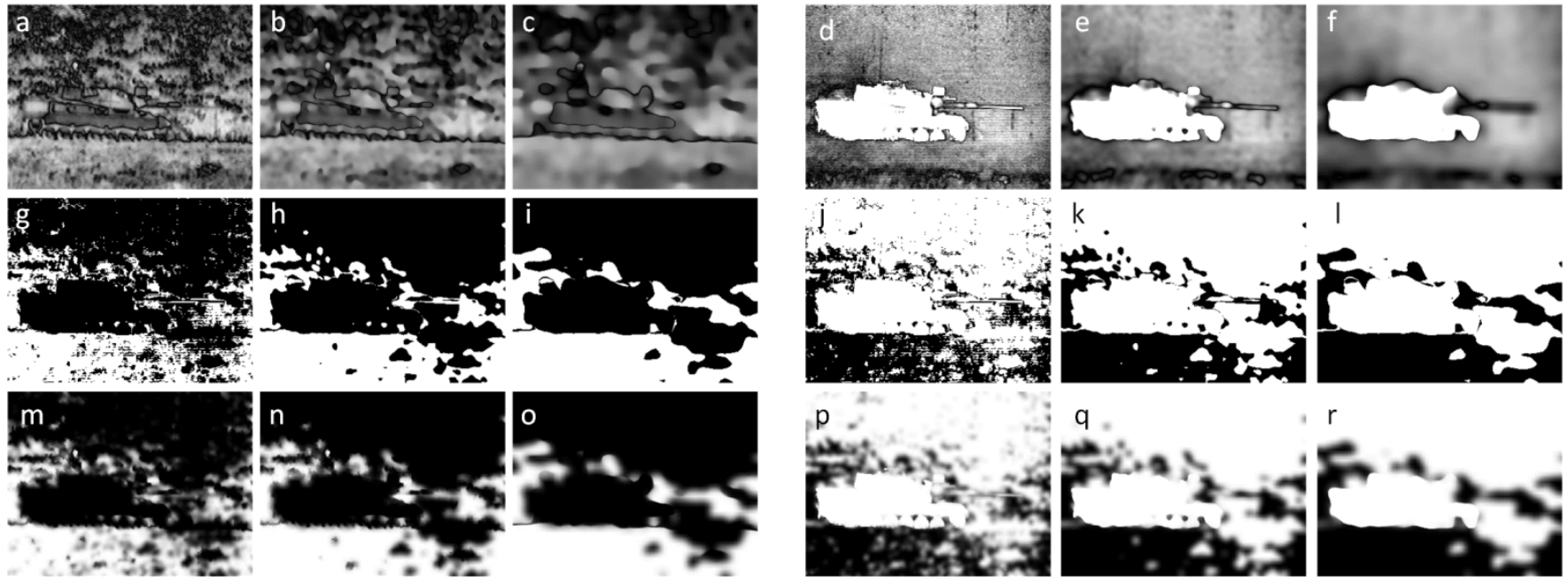

808

809 Figure 4. Computing smoothed weight maps by guided filtering of binary weight maps.

810 Saliency maps at levels 0,1 and 2 for respectively the intensified visual (a-c) and thermal

811 infrared (d-f) images from Figure 3. Complementary binary weight maps for both image

812 modalities (g-i and j-1) are obtained with a pointwise maximum operator at corresponding levels.

813 Smooth continuous weight maps ( $\mathrm{m}-\mathrm{o}$ and $\mathrm{p}-\mathrm{r}$ ) are produced by guided filtering of the binary

814 weight maps with their corresponding base layers as guidance images.

815

816 
817

818
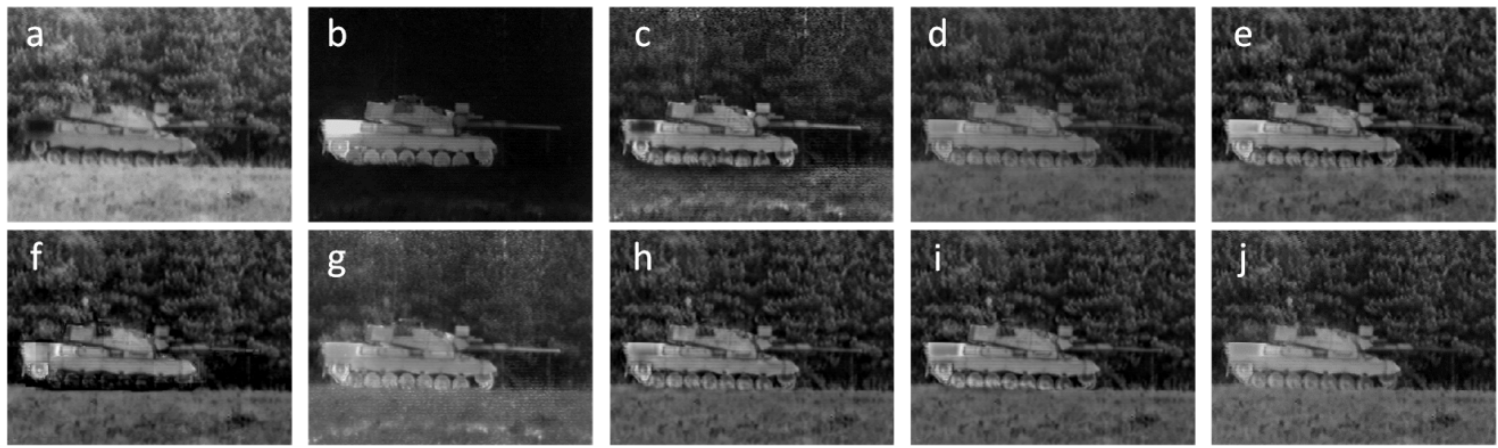

819 Figure 5. Comparison with existing multiresolution fusion schemes.

820 Original intensified visual (a) and thermal infrared (b) images for scene nr 10, and the fused 821 results obtained with respectively a Contrast Pyramid (c), Gradient Pyramid (d), Laplace

822 Pyramid (e), Morphological Pyramid (f), Ratio Pyramid (g), DWT (h), SIDWT (i), and the 823 proposed method (j), for scene nr. 10 .

824

825

826
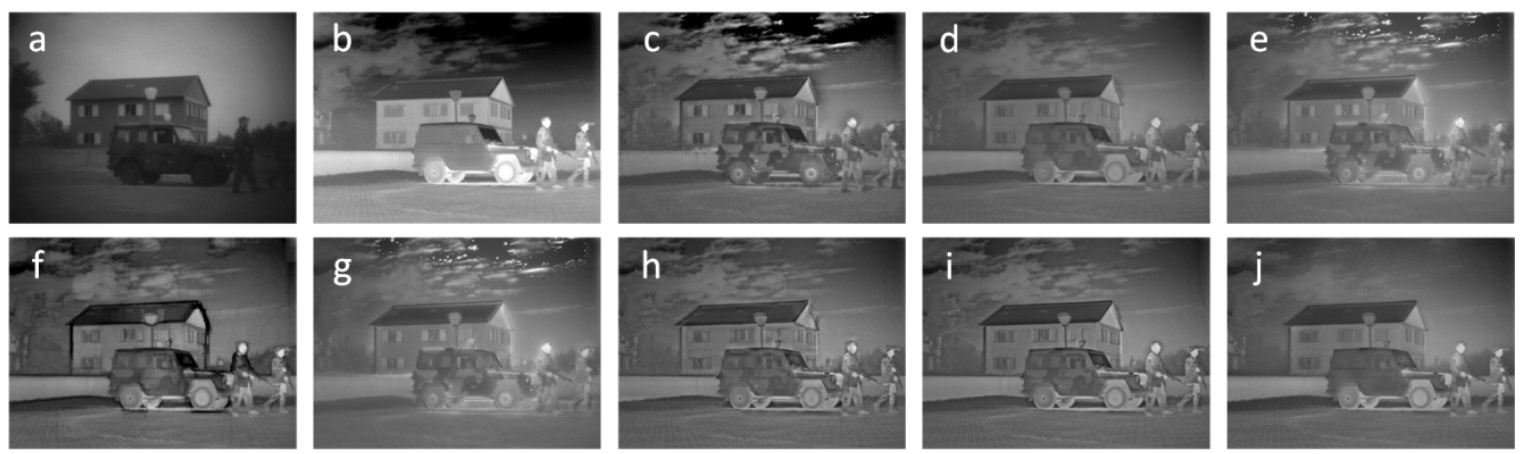

827 Figure 6. As Figure 5, for scene nr. 2.

828

829
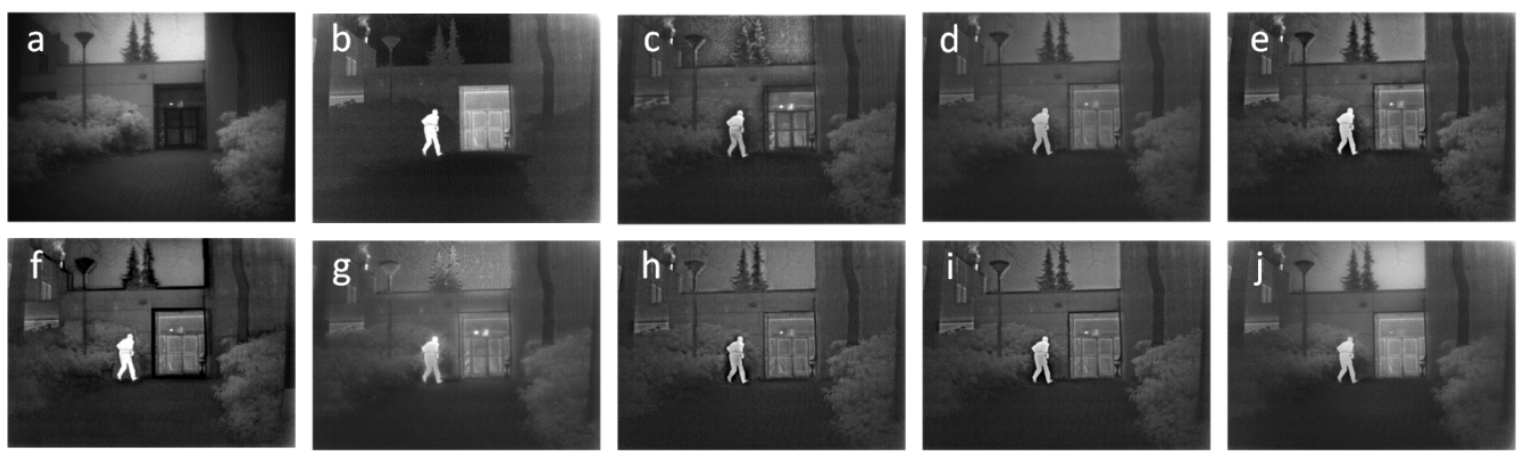

830 Figure 7. As Figure 5, for scene nr. 3. 

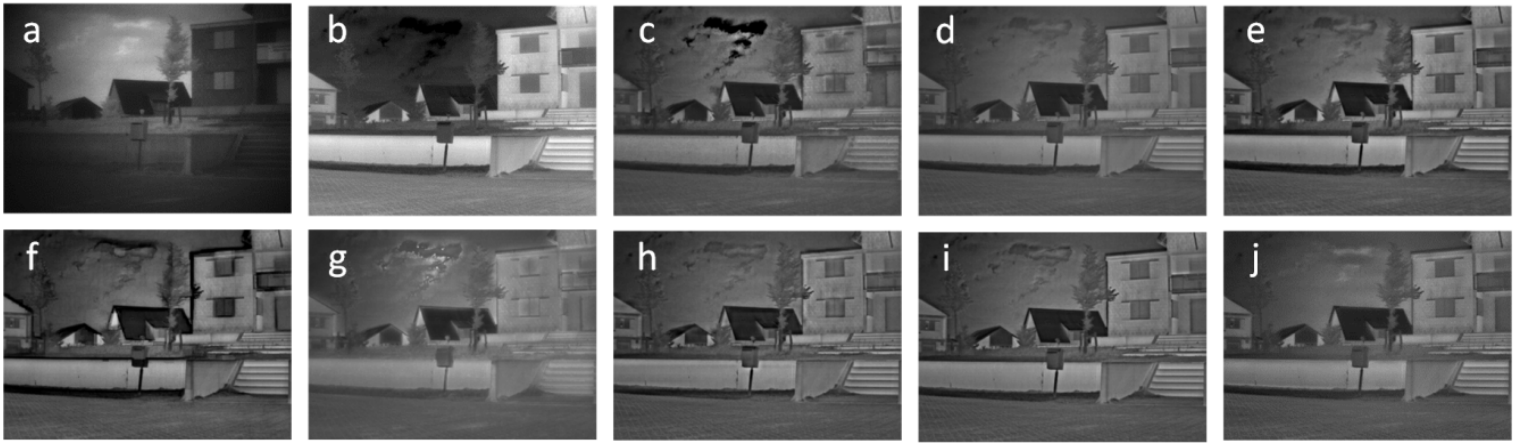

833 Figure 8. As Figure 5, for scene nr. 4.

834

835
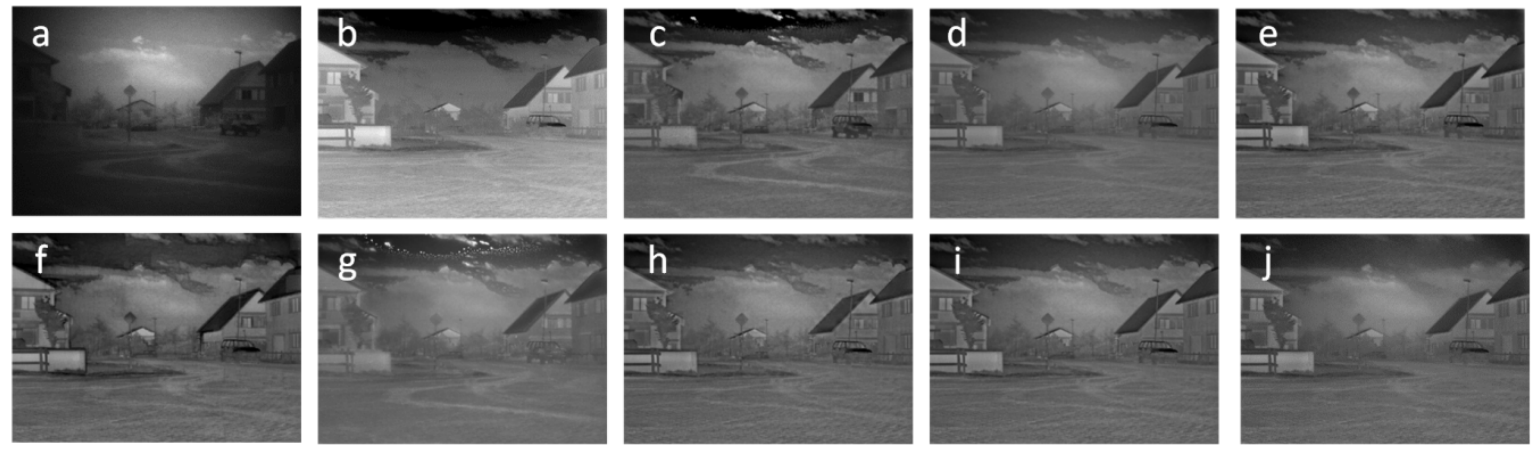

836 Figure 9. As Figure 5, for scene nr. 5.

837

838

839 
840

841 Tables

842

843

844 Table 1. Entropy values for each of the methods tested and for all 12 scenes.

845

\begin{tabular}{cllllllll}
\hline $\begin{array}{c}\text { scene } \\
\text { nr. }\end{array}$ & Contrast & DWT & Gradient & Laplace & Morph & Ratio & SIDWT & IGF \\
\hline 1 & 6.4818 & 6.4617 & 6.1931 & 6.5935 & $\mathbf{6 . 6 9 4 3}$ & 6.5233 & 6.4406 & 6.5126 \\
2 & 6.7744 & 6.6731 & 6.5873 & 6.7268 & 6.9835 & 6.7268 & 6.7075 & $\mathbf{7 . 4 2 3 3}$ \\
3 & 6.4340 & 6.5704 & 6.4965 & 6.6401 & 6.7032 & 6.6946 & 6.5878 & $\mathbf{6 . 8 5 8 9}$ \\
4 & 6.8367 & 6.8284 & 6.6756 & 7.0041 & 7.0906 & 6.7313 & 6.8547 & $\mathbf{7 . 2 4 9 1}$ \\
5 & 6.7549 & 6.6642 & 6.5582 & 6.7624 & 6.8618 & 6.5129 & 6.6813 & $\mathbf{7 . 1 1 7 7}$ \\
6 & 6.3753 & 6.3705 & 6.2430 & 6.5049 & 6.7608 & 6.2281 & 6.4116 & $\mathbf{6 . 9 0 4 4}$ \\
7 & 6.7470 & 6.3709 & 6.1890 & 6.5106 & 6.7445 & 6.3458 & 6.3817 & $\mathbf{6 . 7 8 6 9}$ \\
8 & 6.3229 & 7.3503 & 7.2935 & 7.3794 & 7.3501 & 7.4873 & 7.3406 & $\mathbf{7 . 4 8 9 1}$ \\
9 & 6.4903 & 6.4677 & 6.3513 & 6.5816 & 6.7295 & 6.3306 & 6.4753 & $\mathbf{6 . 7 7 9 6}$ \\
10 & 6.9627 & 7.0131 & 6.8390 & 7.1073 & 7.0530 & 7.0118 & 7.0224 & $\mathbf{7 . 2 7 8 2}$ \\
11 & 6.5442 & 6.4554 & 6.2110 & 6.5555 & $\mathbf{6 . 8 0 5 1}$ & 6.4053 & 6.4572 & 6.2907 \\
12 & 7.3335 & 7.3744 & 7.3379 & 7.3907 & $\mathbf{7 . 4 2 5 1}$ & 7.3486 & 7.3746 & 7.3568 \\
\hline
\end{tabular}

846

847

848 
849 Table 2. MSSIM values for each of the methods tested and for all 12 scenes.

850

\begin{tabular}{cllllllll}
\hline $\begin{array}{c}\text { scene } \\
\text { nr. }\end{array}$ & Contrast & DWT & Gradient & Laplace & Morph & Ratio & SIDWT & IGF \\
\hline 1 & 0.7851 & 0.7975 & 0.8326 & 0.8050 & 0.7321 & 0.8054 & 0.8114 & $\mathbf{0 . 8 3 8 1}$ \\
2 & 0.6018 & 0.6798 & 0.7130 & 0.6406 & 0.6203 & 0.6406 & 0.6935 & $\mathbf{0 . 7 2 1 3}$ \\
3 & 0.7206 & 0.7493 & 0.7849 & 0.7555 & 0.6882 & 0.7468 & 0.7629 & $\mathbf{0 . 7 9 3 2}$ \\
4 & 0.6401 & 0.6790 & 0.7162 & 0.6875 & 0.6155 & 0.6668 & 0.6949 & $\mathbf{0 . 7 1 8 4}$ \\
5 & 0.5856 & 0.6649 & 0.6938 & 0.6695 & 0.6250 & 0.6270 & 0.6769 & $\mathbf{0 . 7 0 3 8}$ \\
6 & 0.5689 & 0.6448 & 0.6755 & 0.6516 & 0.5961 & 0.6099 & 0.6598 & $\mathbf{0 . 6 9 2 1}$ \\
7 & 0.3939 & 0.5742 & 0.5994 & 0.5809 & 0.5320 & 0.4490 & 0.5889 & $\mathbf{0 . 6 3 4 4}$ \\
8 & 0.6474 & 0.6272 & 0.6630 & 0.6392 & 0.5791 & 0.6291 & 0.6463 & $\mathbf{0 . 6 9 4 0}$ \\
9 & 0.6224 & 0.6883 & 0.7224 & 0.6955 & 0.6445 & 0.6718 & 0.7089 & $\mathbf{0 . 7 4 0 5}$ \\
10 & 0.3913 & 0.5410 & 0.5715 & 0.5430 & 0.4899 & 0.4331 & 0.5513 & $\mathbf{0 . 5 9 6 1}$ \\
11 & 0.7174 & 0.7307 & 0.7754 & 0.7439 & 0.6559 & 0.7419 & 0.7539 & $\mathbf{0 . 7 9 0 8}$ \\
12 & 0.7945 & 0.8116 & 0.8466 & 0.8227 & 0.7815 & 0.8106 & 0.8365 & $\mathbf{0 . 8 6 4 6}$ \\
\hline
\end{tabular}

851

852

853 
854

855 Table 3. NMI values for each of the methods tested and for all 12 scenes.

856

\begin{tabular}{cllllllll}
\hline $\begin{array}{c}\text { scene } \\
\text { nr. }\end{array}$ & Contrast & DWT & Gradient & Laplace & Morph & Ratio & SIDWT & IGF \\
\hline 1 & 0.1534 & 0.1692 & 0.2052 & 0.1647 & 0.1699 & 0.1791 & 0.1796 & $\mathbf{0 . 2 8 1 8}$ \\
2 & 0.0989 & 0.0948 & 0.1158 & 0.0897 & 0.1028 & 0.0897 & 0.1028 & $\mathbf{0 . 2 9 9 4}$ \\
3 & 0.0898 & 0.1222 & 0.1493 & 0.1252 & 0.1171 & 0.1320 & 0.1280 & $\mathbf{0 . 2 2 3 1}$ \\
4 & 0.1102 & 0.1097 & 0.1322 & 0.1189 & 0.1169 & 0.1046 & 0.1177 & $\mathbf{0 . 2 2 9 4}$ \\
5 & 0.1236 & 0.1170 & 0.1379 & 0.1252 & 0.1318 & 0.1186 & 0.1251 & $\mathbf{0 . 2 1 6 6}$ \\
6 & 0.0857 & 0.0943 & 0.1162 & 0.0969 & 0.1068 & 0.0902 & 0.0980 & $\mathbf{0 . 2 2 2 9}$ \\
7 & 0.0697 & 0.0711 & 0.0839 & 0.0809 & 0.0888 & 0.0616 & 0.0781 & $\mathbf{0 . 2 1 4 7}$ \\
8 & 0.2192 & 0.1825 & 0.2198 & 0.1832 & 0.1884 & 0.2130 & 0.2021 & $\mathbf{0 . 3 0 9 0}$ \\
9 & 0.0692 & 0.0679 & 0.0781 & 0.0747 & 0.0790 & 0.0690 & 0.0731 & $\mathbf{0 . 2 0 1 3}$ \\
10 & 0.1375 & 0.1643 & 0.2043 & 0.1780 & 0.1761 & 0.1662 & 0.1760 & $\mathbf{0 . 2 9 6 2}$ \\
11 & 0.1055 & 0.1043 & 0.1177 & 0.1100 & 0.1047 & 0.1179 & 0.1115 & $\mathbf{0 . 1 6 4 6}$ \\
12 & 0.2572 & 0.2511 & 0.2746 & 0.2602 & 0.2438 & 0.2660 & 0.2649 & $\mathbf{0 . 2 9 8 7}$ \\
\hline
\end{tabular}

857

858

859

860 
861 Table 4. NFMI values for each of the methods tested and for all 12 scenes.

\begin{tabular}{cllllllll}
\hline $\begin{array}{c}\text { scene } \\
\text { nr. }\end{array}$ & Contrast & DWT & Gradient & Laplace & Morph & Ratio & SIDWT & IGF \\
\hline 1 & 0.4064 & 0.3812 & 0.3933 & 0.3888 & 0.3252 & 0.3498 & $\mathbf{0 . 4 0 8 4}$ & 0.4008 \\
2 & 0.4354 & 0.3876 & 0.4001 & 0.3493 & 0.3432 & 0.3493 & 0.4075 & $\mathbf{0 . 4 3 8 3}$ \\
3 & 0.4076 & 0.4081 & 0.4175 & 0.4138 & 0.3758 & 0.3552 & 0.4330 & $\mathbf{0 . 4 4 5 4}$ \\
4 & 0.4017 & 0.3913 & 0.4066 & 0.4051 & 0.3655 & 0.3497 & 0.4205 & $\mathbf{0 . 4 4 9 0}$ \\
5 & 0.4304 & 0.3971 & 0.4101 & 0.4081 & 0.3758 & 0.3497 & 0.4229 & $\mathbf{0 . 4 5 8 0}$ \\
6 & 0.4299 & 0.4074 & 0.4203 & 0.4164 & 0.3832 & 0.3570 & 0.4295 & $\mathbf{0 . 4 6 0 9}$ \\
7 & $\mathbf{0 . 5 0 5 0}$ & 0.4383 & 0.4439 & 0.4357 & 0.3942 & 0.3779 & 0.4469 & 0.4286 \\
8 & 0.4305 & 0.4074 & 0.4097 & 0.4113 & 0.3806 & 0.3553 & 0.4273 & $\mathbf{0 . 4 3 2 5}$ \\
9 & 0.4351 & 0.3959 & 0.4105 & 0.3995 & 0.3658 & 0.3539 & 0.4130 & $\mathbf{0 . 4 3 7 0}$ \\
10 & 0.4439 & 0.4251 & 0.4263 & 0.4268 & 0.3863 & 0.3465 & 0.4513 & $\mathbf{0 . 5 0 4 5}$ \\
11 & 0.3882 & 0.3798 & 0.3987 & 0.3804 & 0.3131 & 0.3453 & 0.4068 & $\mathbf{0 . 4 2 0 6}$ \\
12 & 0.4051 & 0.3725 & 0.3973 & 0.3820 & 0.3449 & 0.3635 & 0.4111 & $\mathbf{0 . 4 2 5 7}$ \\
\hline
\end{tabular}

862

863

864 